\title{
Lower cognitive test scores at age 7 in children born with marginally low birth weight
}

\author{
Josefine Starnberg ${ }^{1}$, Mikael Norman ${ }^{2}$, Björn Westrup ${ }^{3}$, Magnus Domellöf $^{1}$ and Staffan K Berglund ${ }^{1}$
}

BACKGROUND: Being born with very low birth weight $(<1500 \mathrm{~g})$ is associated with poorer neurocognition later in life. The aim of this study was to explore neurodevelopmental functions in those born with marginally LBW (2000-2500 g).

METHODS: This was originally a randomized controlled trial investigating the effects of early iron supplementation in 285 marginally LBW children. Herein, we explored the combined marginally LBW group and compared their results to 95 normal birth weight (NBW; 2501-4500 g) controls in an observational design. At 7 years, a pediatric psychologist tested the children using Wechsler Intelligence Scale for Children (WISC IV), Beery-Buktenica developmental test of Visual-Motor Integration (Beery VMI), and Test of Everyday Attention for Children (TEA-Ch).

RESULTS: The marginally LBW children had lower verbal comprehension intelligence quotient (IQ) (104 vs. 107, $P=0.004)$, lower VMI scores ( 96.5 vs. $100, P=0.028)$, and lower total mean TEA-Ch scores (8.5 vs. 9.7, $P=0.006$ ), compared to controls. Also, the marginally LBW children group had a higher proportion of children below - 1 SD for VMI and TEA-Ch.

CONCLUSIONS: Marginally LBW children had lower verbal comprehension $I Q$, lower visual-motor integration, and lower attention performance than NBW children, suggesting an increased risk of cognitive difficulties in early school age.

B eing born preterm $(<37$ gestational weeks) or with low birth weight (LBW, $<2500 \mathrm{~g}$ ) is associated with poorer cognitive function, compared to being born at term with normal birth weight (NBW, 2501-4500 g) (1-3). An abundant number of studies have shown that children born with veryLBW (VLBW, $<1500 \mathrm{~g})$ or born extremely preterm $(<28$ gestational weeks) seem to be particularly at risk $(4,5)$ and a negative linear relationship between gestational age at birth or birth weight and later neurocognitive outcomes have been suggested $(3,6-8)$. However, studies including children born moderate-late preterm (32-36 gestational weeks) have displayed more diverging results, and in some studies, the increased risk is no longer observed when adjusting the results for socioeconomic factors $(9,10)$. Consequently, the correlation as well as the clinical relevance is still unclear and recent reviews have emphasized the need of long-term studies and the necessity to study a variety of birth weights and gestational ages (6).

Most children with LBW are born with marginally LBW (2000-2500 g), representing about 3-6\% of all children in developed countries and even more in developing countries $(11,12)$. Despite this being the largest subgroup of LBW children, very little is known about their long-term health $(6,9,13)$. More knowledge is therefore needed to be able to optimize the possibilities for these children to reach their full neurodevelopmental potentials, particularly at school start.

We have previously explored the neurodevelopmental outcomes and the behavior in a cohort of marginally LBW children that were randomized in infancy to different doses of iron supplements. In those, we found higher prevalence of behavioral problems in the placebo group, compared to the iron intervention groups. However, no difference was observed between the iron intervention groups or between the marginally LBW children and controls with regards to intelligence quotient (IQ) at 3.5 or 7 years of age $(14,15)$. In this study, we aimed to assess the capacities of cognition and attention in the combined cohort of marginally LBW children at 7 years of age, using an observational design. We assessed the LBW children with three different psychological tests and compared their results to those of peers born with NBW.

\section{METHODS}

Study Design and Participants

This was a prospective observational study including 285 children born between March 2004 and November 2007, at tertiary hospitals in Stockholm and Umeå, Sweden. The marginally LBW infants were identified from delivery records and were recruited at 6 weeks of age. Inclusion criteria were: being born with a birth weight between 2000 and $2500 \mathrm{~g}$, not having a chronic disease, and not having been treated with blood transfusion or iron supplements. Children with diagnosed congenital disorders that might affect cognition were excluded in all analyses, whereas children who were diagnosed with blood disorders or anemia (hemoglobin $<90 \mathrm{~g} / \mathrm{l}$ ) at inclusion were excluded when comparing the intervention groups (16).

The iron intervention has been described in detail elsewhere (16). Briefly, the children were stratified at inclusion according to sex and study center, and by computerized randomization, they were

\footnotetext{
'Department of Clinical Sciences, Pediatrics, Umeå University, Umeå, Sweden; ${ }^{2}$ Division of Pediatrics, Department of Clinical Science, Intervention and Technology, Karolinska Institutet, Stockholm, Sweden; ${ }^{3}$ Division of Neonatology, Department of Women's and Children's Health, Karolinska Institutet, Stockholm, Sweden. Correspondence: Josefine Starnberg (josefine.starnberg@umu.se)

Received 29 August 2017; accepted 19 January 2018; advance online publication 2 May 2018. doi:10.1038/pr.2018.35
} 


\section{Articles | Starnberg et al.}

allocated into three double-blinded intervention groups receiving placebo, $1 \mathrm{mg}$ iron/kg per day, or $2 \mathrm{mg}$ iron $/ \mathrm{kg}$ per day, respectively. The intervention continued between 6 weeks and 6 months of age and the dose was adjusted for the child's weight at 12 and 19 weeks of age. The drops of placebo, prepared by Apoteket Production \& Laboratories (Stockholm, Sweden), and of iron supplementation (ferrous succinate drops of Ferromyn S; Astra Zeneca, Södertälje, Sweden) were of similar taste and color. The parents were given identical bottles and were instructed to administer the dose between meals using syringes.

At 3.5 years, a group of 95 control children were enrolled. To do this, every third born marginally LBW child was identified and selected as the index case. Each index case was used to make a list of 10 children who were born closest in time at the same study center, and fulfilling the inclusion criteria: born with a birth weight between 2501 and $4500 \mathrm{~g}$, born at gestational week $37+0-42+0$, not having been admitted to neonatal ward, and not having been diagnosed with any congenital disease. The parents to the child born closest in time to the index case were contacted and asked to join the study. If they declined, the next child's parents on the list were contacted, until all selected index cases had a matching control child.

Parents signed a written consent form when included in the study. This study was approved by the regional ethical review boards in Stockholm and Umeå. The study is registered at Clinicaltrials.gov (Iron Supplementation of Marginally Low Birth Weight Infants (John)).

\section{Data Collection}

At inclusion, background (parental education, smoking habits, and country of birth) and baseline data (birth weight, birth length, head circumference, gestational age at birth, delivery means, Apgar score, and neonatal diagnoses) were assessed from the parents and from the delivery records. Standard deviation score for length and weight were calculated using a sex- and age-specific growth standard (17). Small for gestational age was defined, as standard deviation score for birth weight $<-2$ and all other cases were considered appropriate for gestational age. Being born preterm was defined as born $<37+0$ gestational weeks, whereas the children born $\geq 37+0$ gestational weeks were defined as term. Except for the iron or placebo supplementation as part of the original intervention study, all children were evaluated individually and received equal care according to standard health-care programs at each site. At 7 years of age, the children were examined based on the anthropometric data and an experienced authorized pediatric psychologist evaluated the cognitive performance using the Swedish version of the three validated tests: Wechsler Intelligence Scale for Children-fourth edition (WISC IV), Test of Everyday Attention for Children (TEA$\mathrm{Ch}$ ), and Beery-Buktenica developmental test of Visual-Motor Integration (Beery VMI). Using an individual coding system handled by the study nurse, the psychologist was blinded regarding group belonging (LBW or not). Furthermore, parents were asked not to reveal any information regarding the perinatal weight or the gestational age to the psychologist.

WISC is a validated test for intellectual ability in children aged 616 years of age. The test consists of four domains: verbal comprehension, perceptual reasoning/visual-spatial index, working memory, and processing speed/fluid reasoning, as well as the four domains combined in a full-scale index. The results were registered as raw points and directly converted into age-specific scale points and IQ, where 100 is mean and SD is 15 in a reference population (18). To further explore the differences between the groups, cases with IQ below $85(<-1 \mathrm{SD})$ were identified in dichotomized variables.

Beery VMI is a test for visual perception and motor integration. In this test, the child's task is to copy 24 figures by hand, thereby assessing the ability to coordinate visual perception and hand-finger movements (19). This test correlates well with school achievement, foremost mathematics, and reading (20). The test results are registered as raw points and then converted into age-specific standard scores, where 100 is mean and SD is 15 in a reference population. Standard score below $85(<-1 \mathrm{SD})$ was used as cut off when dichotomizing the scores of VMI.

TEA-Ch is a test for everyday attention. The test is divided into nine different sections, of which we used the first two parts (four subtests in total) to briefly explore different attention capacities. The first test, sky search, is a test for selective attention, in which the child's task is to identify and mark targets on a sheet with distractors. From this task, the child gets points for the targets found, time per target, and overall attention, the latter of the possible influence of motor control has been subtracted from the results. The second part is score!, in which the child listens to and counts the tunes, thereby assessing the child's ability to sustain his or hers attention to perform a task, removing the influence of motor control. The scores were registered as raw scores and converted into age- and sex-specific scale scores based on a reference population with a mean (SD) of 10 (3). An individual mean TEA-Ch score was calculated as the mean scale score for the four subtests. For dichotomized analyses, scale scores below $7(<-1 \mathrm{SD})$ were used as cutoffs.

\section{Statistical Analyses}

The power calculation was based on neurocognitive outcomes. Assuming a dropout rate of $20 \%$, poor compliance rate of $15 \%$, aiming at a power of $80 \%$, and a significance level of 0.05 , an effect size of 0.5 SD (7.5 IQ scores) would be detectable $(14,16)$.

SPSS 23.0 for Windows (SPSS Inc, Chicago, IL) was used for all the analyses. Parental and perinatal characteristics, as well as studied outcomes, were compared between the marginally LBW group and the control group, using Student's independent $t$ test for continuous variables and $\chi^{2}$ test for proportions. Following unadjusted analyses, the studied outcomes were also compared between the groups in confounder adjusted analyses using analyses of covariance. We adjusted for preselected confounders (sex of the child, mother's ethnicity, and age at child birth, as well as parental education), previously described as important sociodemographic factors $(4,22)$. In addition, we also stratified the marginally LBW children for being born preterm or term and compared each subgroup to the controls in all analysed outcomes. When comparing the test results among the original randomization groups for the possible effects by early iron supplements, ANOVA was performed.

\section{RESULTS}

Figure 1 presents the study flow chart. Two hundred and eighty-five marginally LBW children were included in the study at 6 weeks of age with an addition of $95 \mathrm{NBW}$ control children recruited at 3.5 years of age. Three children were excluded in all analyses due to congenital disorders. At 7 years of age, 219 marginally LBW children (77\%) and 74 control children $(78 \%)$ responded to the invitation to participate. However, 18 marginally LBW children and 4 control children did not complete the psychological evaluations and were not included in any of the present analyses. The follow-up of the original intervention study had been reported previously (15). There was no difference in the reported morbidity or side effects between the intervention groups (16).

As shown in Table 1, the marginally LBW children had a significantly lower proportion of mothers being born in Scandinavia, compared to controls ( $82 \%$ vs. $94 \%, P=0.017)$. As expected, the control group had a higher proportion of vaginal deliveries ( $73 \%$ vs. $57 \%, P=0.031)$, higher proportion of singleton births (99\% vs. $71 \%, P=0.001$ ), and higher Apgar score at $5 \mathrm{~min}(9.8$ vs. $9.4, P=0.013)$ than the marginally 
Neurocognition after low birth weight $\mid$ Articles

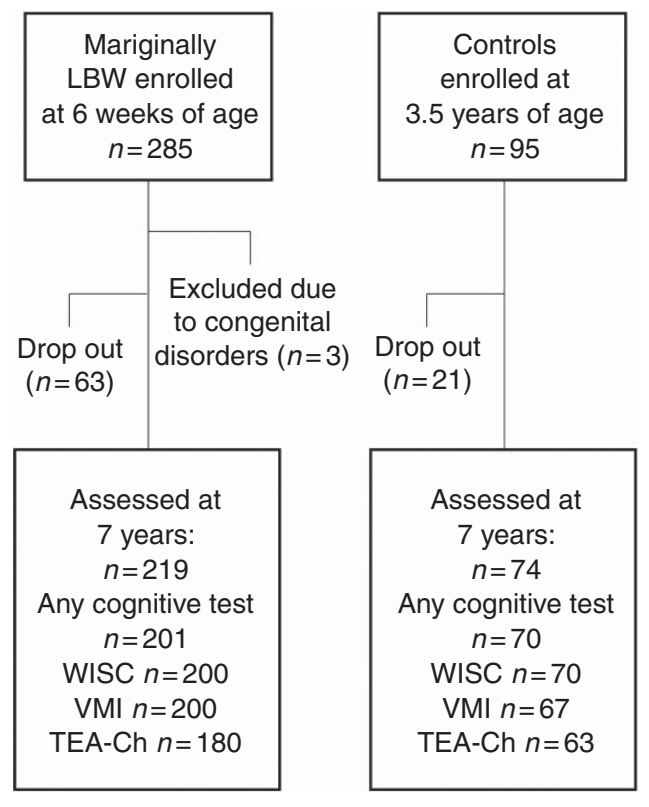

Figure 1. Flow chart of 285 children included marginally low birth weight children (marginally LBW; 2000-2500 g) and 95 normal birth weight children (NBW; 2501-4500 g).

LBW children. The marginally LBW children were by definition smaller in size at birth.

We have recently shown that the WISC IV scores (IQ) did not differ among the intervention groups at 7 years (15). Also, as presented in Supplementary Table S1, the iron intervention had no significant effect on VMI or TEA-Ch scores at 7 years. Therefore, the three intervention groups were combined in the following analyses.

As presented in Table 2, the marginally LBW children had significantly lower scores than the NBW controls in unadjusted analyses for verbal comprehension IQ, VMI, and all the TEA-Ch subtests except for score! In a multivariate model adjusting for potential confounders, scores remained significantly lower in marginally LBW children for verbal comprehension IQ $(P=0.004)$, VMI $(P=0.028)$, and the subtests for selective attention (targets found $P=0.018$ and time per target $P=0.018$ ), as well as mean TEA-Ch $(P=0.006)$.

In the stratified analyses, comparing term and preterm infants separately to controls, we observed that both children born preterm and term had generally lower scores than controls in all neurocognitive tests, except in the WISC domain of processing speed. In the confounder-adjusted statistical analyses, these differences reached significance for preterm born children $(n=110)$ with regards to verbal comprehension IQ and three out of four subtests of TEA$\mathrm{Ch}$, as well as in mean TEA-Ch scores. The marginally LBW children born at term $(n=90)$ had significantly lower scores at the VMI test and in one of the TEA-Ch subtests, compared to controls.

In Table 3, we further explored the test results of WISC IV, VMI, and TEA-Ch by identifying children below $-1 \mathrm{SD}(<85$
Table 1. Selected characteristics of the 70 included children born with NBW (2501-4500 g) and 201 children born with marginally LBW (2000-2500 g)

\begin{tabular}{|c|c|c|c|}
\hline & $\begin{array}{c}\text { Controls, } \\
n=70\end{array}$ & $\begin{array}{c}\text { Marginally LBW, } \\
n=201\end{array}$ & $P$ value \\
\hline \multicolumn{4}{|l|}{ Parental characteristics } \\
\hline $\begin{array}{l}\text { Maternal age at child } \\
\text { birth }\end{array}$ & $32.4(4.4)$ & $32.2(4.8)$ & 0.776 \\
\hline $\begin{array}{l}\text { Mother born in } \\
\text { Scandinavia }\end{array}$ & $65(94 \%)$ & $165(82 \%)$ & 0.017 \\
\hline $\begin{array}{l}\text { Mother educated at } \\
\text { university }\end{array}$ & 39 (57\%) & 119 (63\%) & 0.468 \\
\hline $\begin{array}{l}\text { Father educated at } \\
\text { university }\end{array}$ & $36(54 \%)$ & 107 (60\%) & 0.659 \\
\hline $\begin{array}{l}\text { Smoking during } \\
\text { pregnancy }\end{array}$ & $2(3 \%)$ & $6(3 \%)$ & $>0.999$ \\
\hline Mother's BMI $\left(\mathrm{kg} / \mathrm{m}^{2}\right)$ & $23.5(3.6)$ & $24.0(4.6)$ & 0.457 \\
\hline Father's BMI $\left(\mathrm{kg} / \mathrm{m}^{2}\right)$ & $25.3(3.2)$ & $25.4(3.1)$ & 0.893 \\
\hline
\end{tabular}

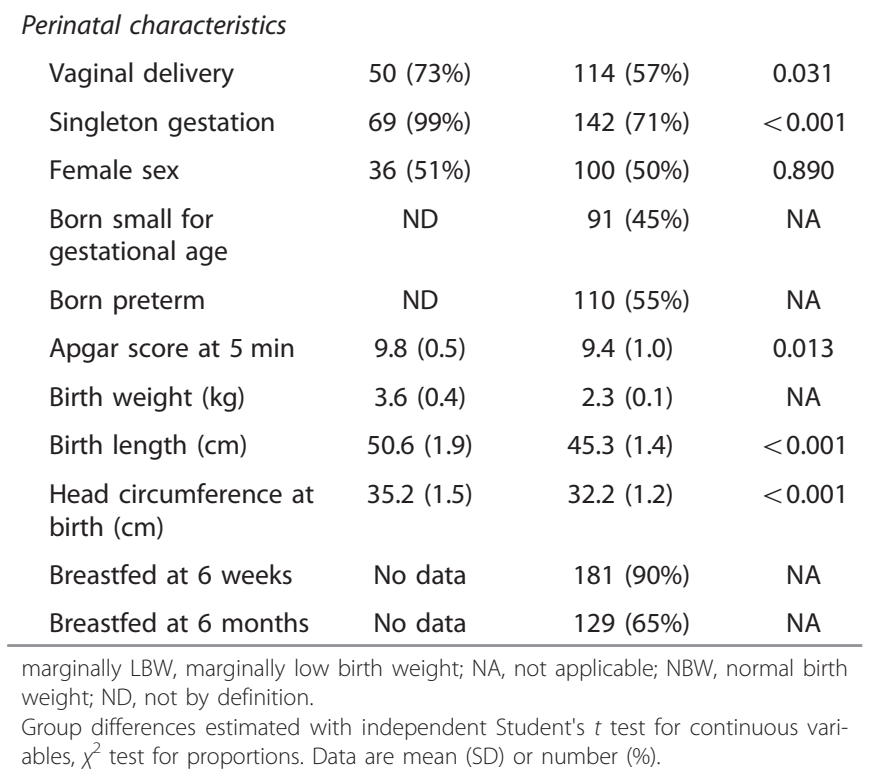

for WISC IV and VMI, $<7$ for TEA-Ch) and compared the proportions between the LBW children and the controls. In these, there were no significant differences when comparing the IQ domains, regardless of being born term or preterm. However, compared to the NBW children, marginally LBW children had higher proportions below - 1 SD for VMI (14\% vs. $5 \%, P=0.045$ ), three of the TEA-Ch subtests (targets found, $16 \%$ vs. $3 \%, P=0.008$; time per target, $52 \%$ vs. $30 \%$, $P=0.003$, and attention score, $38 \%$ vs. $24 \%, P=0.046$ ), as well as mean TEA-Ch scores $(27 \%$ vs $14 \%, P=0.040)$. When stratifying these analyses for being born preterm or term, the prevalence of low VMI scores remained significant in the term subgroup of marginally LBW children, compared to controls $(P=0.022)$, whereas in TEA-Ch, there were significantly higher prevalence in both preterm and in term born LBW children, compared to controls. 


\section{Articles | Starnberg et al.}

Table 2. Group differences for WISC IV, Beery VMI, and TEA-Ch between NBW children (2501-4500 g) and marginally LBW children $(2000-2500 \mathrm{~g})$ at 7 years of age, including analyses stratified for being born term or preterm

\begin{tabular}{|c|c|c|c|c|}
\hline & \multirow[b]{2}{*}{ Controls } & \multirow[t]{2}{*}{ Marginally LBW (all) } & \multicolumn{2}{|c|}{ Marginally LBW subgroups } \\
\hline & & & Preterm & Term \\
\hline WISC & $n=69-70$ & $n=197-200$ & $n=107-110$ & $n=90$ \\
\hline Perceptual reasoning IQ & $106.8(11.2)$ & $105.0(11.6)$ & $106.3(11.5)$ & $103.5(11.6)$ \\
\hline Working memory IQ & $88.8(10.2)$ & $87.4(12.6)$ & $87.1(12.5)$ & $87.7(12.7)$ \\
\hline Beery VMI & $n=67$ & $n=200$ & $n=109$ & $n=91$ \\
\hline Standardized score & $100.0(11.2)$ & $96.5(11.8)^{a, b}$ & $98.2(12.4)$ & $94.4(10.8)^{a, b}$ \\
\hline TEA-Ch test & $n=57-63$ & $n=158-180$ & $n=85-98$ & $n=73-83$ \\
\hline Score! & $10.2(3.3)$ & $9.12(3.7)$ & $8.99(3.6)^{a}$ & $9.27(3.9)$ \\
\hline Mean TEA-Ch & $9.73(2.2)$ & $8.49(2.8)^{a, b}$ & $8.37(2.5)^{a, b}$ & $8.63(3.1)^{\mathrm{a}}$ \\
\hline
\end{tabular}

ANCOVA, analyses of covariance; Beery VMI, Beery-Buktenica developmental test of Visual-Motor Integration; IQ, intelligence quotient; marginally LBW, marginally low birth weight; NBW, normal birth weight; TEA-Ch, Test of Everyday Attention for Children; WISC IV, Wechsler Intelligence Scale for Children.

Data are mean (SD).

${ }^{a} P$ value $<0.05$, for differences between marginally LBW children and controls using independent Student's $t$ test.

${ }^{\mathrm{b}} \mathrm{P}$ value $<0.05$ adjusted for sex, maternal age, whether mothers are born in Scandinavia, maternal education level, fathers level of education, using ANCOVA.

\section{DISCUSSION}

This study demonstrates three important findings: first, we found significantly lower cognitive scores in marginally LBW children than in NBW children for all the three validated psychological tests used herein. Second, we also found larger proportions of marginally LBW children with suboptimal cognitive performance, compared to NBW children. Finally, the lower cognitive scores were observed in both preterm and in term born marginally LBW children, suggesting that the neurocognitive differences are independent of gestational age at birth. All together, these observations suggest an increased risk of poorer cognitive performance in this large but less explored subgroup of LBW children, representing a significant proportion of all children.

Our finding that children born only with marginally LBW had lower verbal comprehension IQ than NBW peers indicates partially cognitive difficulties. This is in agreement with several previous studies of very-LBW $(<1500 \mathrm{~g})$ or extremely preterm $(<28$ gestational weeks) children, but has rarely been shown in this particular subgroup $(4,5,8)$. In contrast to our findings, two studies that compared moderate-late preterm with children born at term did not observe any differences in verbal IQ, but in other domains of intelligence $(22,23)$. Notable is that we did not detect any differences between marginally LBW children and controls in the first neurocognitive follow-up at 3.5 years (14). Whether this association extends to or becomes even more marked in adulthood remains to be studied. However, according to a meta-analysis by Kormos et al. (10), adults born with LBW had 7.6 lower IQ (full scale) than peers born with NBW, suggesting that the neurodevelopmental consequences could persist throughout life. The effect size (3 IQ points) is in similarity with the differences observed between breast-fed and formula-fed infants and is most likely of clinical significance (24).

Beery VMI is a test that correlates well to school achievements (20). Consequently, our observation of significantly lower VMI scores in the marginally LBW born children suggests an increased risk of school difficulties for this large but otherwise healthy group of children. Again, previous studies have shown similar results in very LBW children. Geldof et al. (25) found that visual-motor integration in very-LBW children were significantly poorer than in NBW peers. With regard to children born with marginally LBW, we have not identified any other study exploring the effects on VMI, suggesting that our findings are novel.

The TEA-Ch test is a less explored measure in preterm and LBW children, and again, we have not been able to detect any former reports of TEA-Ch in marginally LBW children. According to our results, the marginally LBW children had significantly lower scores than NBW peers in almost all subtests of TEA-Ch. Attention is a complex concept and TEA-Ch is a test aiming to explore the different attention capacities in the child, including selective and sustained attention. According to our results, marginally LBW children have more difficulties in selective attention than NBW controls, especially in those subtests allowing for motor 
Table 3. Cognitive outcome below - 1 SD in 7-year-old children born with marginally LBW (2000-2500 g) compared to children born with NBW (2501-4500 g), including stratified analyses for preterm and term born children

\begin{tabular}{|c|c|c|c|c|}
\hline & \multirow[b]{2}{*}{ Controls } & \multirow{2}{*}{$\begin{array}{l}\text { Marginally } \\
\text { LBW (all) }\end{array}$} & \multicolumn{2}{|c|}{$\begin{array}{l}\text { Marginally LBW } \\
\text { subgroups }\end{array}$} \\
\hline & & & Preterm & Term \\
\hline \multicolumn{5}{|l|}{ WISC IV $<85$} \\
\hline $\begin{array}{l}\text { Verbal } \\
\text { comprehension IQ }\end{array}$ & $1(1 \%)$ & $4(2 \%)$ & $2(2 \%)$ & $2(2 \%)$ \\
\hline $\begin{array}{l}\text { Perceptual reasoning } \\
\text { IQ }\end{array}$ & $2(3 \%)$ & 7 (4\%) & $2(2 \%)$ & $5(6 \%)$ \\
\hline Working memory IQ & $24(35 \%)$ & $95(48 \%)$ & $50(46 \%)$ & $45(50 \%)$ \\
\hline Processing speed IQ & $9(13 \%)$ & $28(14 \%)$ & $16(15 \%)$ & $12(13 \%)$ \\
\hline Full scale IQ & $3(4 \%)$ & $13(6 \%)$ & 9 (8\%) & $4(4 \%)$ \\
\hline
\end{tabular}

VMI $<85$

Standard score $\quad 3(5 \%) \quad 28(14 \%)^{\mathrm{a}} \quad 13(12 \%) \quad 15(17 \%)^{\mathrm{a}}$

TEA-Ch $<7$

$\begin{array}{lcccc}\begin{array}{l}\text { Sky search, targets } \\ \text { found }\end{array} & 2(3 \%) & 28(16 \%)^{\mathrm{a}} & 12(12 \%) & 16(20 \%)^{\mathrm{a}} \\ \begin{array}{l}\text { Sky search, time } \\ \text { per target }\end{array} & 19(30 \%) & 93(52 \%)^{\mathrm{a}} & 54(55 \%)^{*} & 39(48 \%)^{\mathrm{a}} \\ \begin{array}{l}\text { Sky search, } \\ \text { attention score }\end{array} & 15(24 \%) & 67(38 \%)^{\mathrm{a}} & 37(38 \%) & 30(37 \%) \\ \begin{array}{l}\text { Score! } \\ \text { Mean TEA-Ch }\end{array} & 8(14 \%) & 40(25 \%) & 23(27 \%) & 17(23 \%) \\ \end{array}$

Beery VMI, Beery-Buktenica developmental test of Visual-Motor Integration; IQ, intelligence quotient; marginally LBW, marginally low birth weight; NBW, normal birth weight; TEA-Ch, Test of Everyday Attention for Children; WISC IV, Wechsler Intelligence Scale for Children.

Data are number (\%) of identified cases below average.

a $P$ value $<0.05$, for differences of proportions between the groups, using $x^{2}$ test, Fisher's exact when $<5$ cases in any group.

control influence. In a study by Anderson et al. (1), children born extremely preterm or with extremely LBW experienced the same difficulties with regard to TEA-Ch at 8 years of age. In addition, in a review by de Jong et al. (13), also moderate to late preterm children had more attention problems, compared to those born term.

Following several previous studies of neurodevelopment in preterms, there is an established evidence that preterm birth and LBW constitutes a risk factor for later attention problems, poorer cognitive development, and school difficulties, compared to children born at term with NBW $(2,13)$. The mechanisms behind this association are most likely correlated to the immature brain following preterm birth. It has been suggested that morphological disturbances in the preterm infants leave permanent regional changes in the structure of the brain, remaining until later childhood $(6,26)$. In a neuroimaging study by Peterson et al. (27), preterm born children had smaller regional cortical brain volumes in sensorimotor and midtemporal cortex, at 8 years of age.
The authors found that the volumes of these cortical regions were associated with later verbal, performance, and full-scale IQ (domains of an earlier version of the WISC test). In addition, they correlated VMI test results to regional volumes in the brain and found several interesting correlations, of whom many were also affected by preterm birth, supporting a causality to premature brain morphology. To further support this model, it has previously been shown that at 6-8 years of age, sensorimotor performance was associated with lower IQ in preterms, an association that could not be seen in term born children (28).

The present study was originally designed to explore if iron deficiency may contribute to the impaired neurocognitive performance in LBW children. We have previously found that there was a positive effect of early iron intervention on behavior at 3.5 and at 7 years $(14,15)$. However, no effect was observed on the cognitive tests WIPSII III at 3.5 years or WISC IV test at 7 years. Herein, we further showed that when exploring the outcomes of VMI and TEA-Ch, there were no differences between the iron intervention groups. Thus, it is unlikely that iron deficiency in infancy contributes to the observed neurocognitive differences.

To explore whether our observed differences between marginally LBW and NBW children was linked to the high proportion of preterm birth, we stratified our analyses. Surprisingly, we found that both preterm and term born LBW children had lower verbal comprehension IQ scores, indicating that both subgroups of marginally LBW children are at a risk of lower verbal intelligence. Further supportive to this hypothesis is our observation that the term born children, and not the preterms, had significantly poorer results in VMI. Consequently, it is likely that the mechanism behind the cognitive impairments in marginally LBW born children relates to other mechanisms than only gestational age at birth. Of note is that $84 \%$ of the term born children in our study were born small for gestational age. Owing to the lack of prenatal data, the causes for being born preterm or small for gestational age have not been further evaluated. In other reports, children born small for gestational age, and especially those exposed to intrauterine growth restriction, are more prone to cognitive disadvantages than children born appropriate for gestational age (29). In similarity with verbal comprehension IQ, both term and preterm born children presented lower scores in TEA-Ch than the control group, further supporting our hypothesis that other mechanisms, other than preterm birth, contribute to these impairments

To further explore our findings, we used the test-specific reference populations to identify children with a neurocognitive score below $-1 \mathrm{SD}$. Despite the significant differences in verbal comprehension IQ between marginally LBW and NBW children, there were no differences when comparing proportions of cases below $-1 \mathrm{SD}$. Notable is that both our observed groups presented a verbal comprehension IQ above the reference mean (100) and very low proportions of cases $<-1 \mathrm{SD}$, implying that the observed group differences may not be of much clinical significance for the individual, but 


\section{Articles | Starnberg et al.}

might still be important on a population level. Of further note is that both groups performed well on all tests, except for the domain working memory, in which $\sim 50 \%$ in all groups had IQ $<-1$ SD. This was probably due to a limitation of the Swedish version of the test, and has been noted in other reports as well (8). In contrast to the results from WISC tests, we found that the proportions of children below - 1SD for VMI was significantly higher in the marginally LBW group. This observation was more prominent in the subgroup born at term, indicating that this subgroup of marginally LBW children has more clinically relevant difficulties in visualmotor integration. Also, when exploring the dichotomized results of the TEA-Ch test, there were higher proportions of marginally LBW children scoring $<-1 \mathrm{SD}$. The difference was observed in both the preterm and the term subgroups of marginally LBW children, suggesting that marginally LBW children might have more clinically relevant difficulties in selective attention than NBW peers at 7 years of age, regardless of being born preterm or term. In summary, our dichotomized analyses suggest that the difference between marginally LBW and NBW children does not only consist of differences in mean values but also a significantly increased risk of having a cognitive score below a clinically relevant cutoff. This may have large child health implications.

This study had an observational design applied to a previous randomized double-blinded trial, which limits our findings. Furthermore, the children were recruited from Stockholm and Umeå, two university cities, which might limit the external generalizability to the population. However, the subjects belonged to a well-defined population, the control group was carefully matched, and the study followed a longitudinal design, all strengthening the reliability of our findings. An additional strength was that the psychologists acting test leaders were blinded to the groups.

\section{CONCLUSION}

This study showed that otherwise healthy marginally LBW children born in Sweden presents lower verbal comprehension IQ and lower scores in Beery VMI, as well as lower scores in TEA-Ch than NBW children, even after adjusting for confounders. These are novel findings that we believe will fill the gap of knowledge within the field. Also, the results are of a surprisingly large effect size, suggesting that more studies with long-term follow-up even beyond childhood, as well as testing a wide aspect of cognition, are needed. Our results suggest that marginally LBW children would benefit from neurocognitive screening at school start, to identify cases in need of special support.

\section{SUPPLEMENTARY MATERIAL}

Supplementary material is linked to the online version of the paper at http://www.nature.com/pr

\section{ACKNOWLEDGMENTS}

We thank all participating families, our research nurses Kerstin Andersson and Åsa Sundström, and our pediatric psychologists Lea Forsman and Stephanie Sundén-Cullberg, Anna Crüsell. and Marie Adamsson Johansson.

\section{STATEMENT OF FINANCIAL SUPPORT:}

This work was supported by grants from the Swedish Research Council Formas (grant 222-2002-1894), the Swedish Research Council for Health, Working Life and Welfare (grant FORTE-2012-0708), the Swedish Heart Lung Foundation (project 20090380), the Jerring Foundation, the Oskar Foundation, the Childhood Foundation of the Swedish Order of Freemasons, a regional agreement between Umeå University and Västerbotten County Council (ALF), and a regional agreement on clinical research (ALF) between Stockholm County Council and Karolinska Institutet.

Disclosure: The authors declare no conflict of interest.

\section{REFERENCES}

1. Anderson PJ, De Luca CR, Hutchinson E, et al. Attention problems in a representative sample of extremely preterm/extremely low birth weight children. Dev Neuropsychol 2011;36:57-73.

2. Aarnoudse-Moens CS, Weisglas-Kuperus N, van Goudoever JB, et al. Meta-analysis of neurobehavioral outcomes in very preterm and/or very low birth weight children. Pediatrics 2009;124:717-28.

3. Bhutta AT, Cleves MA, Casey PH, et al. Cognitive and behavioral outcomes of school-aged children who were born preterm: a metaanalysis. JAMA 2002;288:728-37.

4. Farooqi A, Adamsson M, Serenius F, et al. Executive functioning and learning skills of adolescent children born at fewer than 26 weeks of gestation. PLoS ONE 2016;11:e0151819.

5. Pyhälä R, Lahti J, Heinonen $\mathrm{K}$, et al. Neurocognitive abilities in young adults with very low birth weight. Neurology 2011;77:2052.

6. Baron IS, Litman FR, Ahronovich MD, et al. Late preterm birth: a review of medical and neuropsychological childhood outcomes. Neuropsychol Rev 2012;22:438-50.

7. Yang S, Platt RW, Kramer MS. Variation in child cognitive ability by week of gestation among healthy term births. Am J Epidemiol 2010;171: 399-406.

8. Serenius F, Ewald U, Farooqi A, et al. Neurodevelopmental outcomes among extremely preterm infants 6.5 years after active perinatal care in Sweden. JAMA Pediatr 2016;170:954-63.

9. Jennifer DB, Elaine MB. Born just a few weeks early: does it matter? Arch Dis Child Fetal 2013;98:F85-8.

10. Kormos CE, Wilkinson AJ, Davey CJ, et al. Low birth weight and intelligence in adolescence and early adulthood: a meta-analysis. J Public Health (Oxf) 2014;36:213-24.

11. UNICEF. United Nations Children's Fund and World Health Organization, Low birth weight: Country, regional and global estimates. New York: UNICEF, 2004.

12. Graviditeter, förlossningar och nyfödda barn: medicinska födelseregistret 1973-2014: assisterad befruktning 1991-2013. Socialstyrelsen: Stockholm, 2015.

13. de Jong M, Verhoeven M, van Baar AL, et al. School outcome, cognitive functioning, and behaviour problems in moderate and late preterm children and adults: a review. Semin Fetal Neonatal Med 2012;17:163-9.

14. Berglund SK, Westrup B, Hägglöf B, et al. Effects of iron supplementation of LBW infants on cognition and behavior at 3 years. Pediatrics 2013;131: 47.

15. Berglund SK, Chmielewska A, Starnberg J, et al. Effects of iron supplementation of low-birth-weight infants on cognition and behavior at 7 years: a randomized controlled trial. Pediatr Res 2018;83:111-8.

16. Berglund S, Westrup B, Domellof M. Iron supplements reduce the risk of iron deficiency anemia in marginally low birth weight infants. Pediatrics 2010;126:e874-83.

17. Niklasson A, Ericson A, Fryer JG, et al. An update of the Swedish reference standards for weight, length and head circumference at birth for given gestational age (1977-1981). Acta Paediatr Scand 1991;80:756-62.

18. Wechsler D. Weschsler Intelligence Scale for Children-Fourth Edition (WISC-IV). San Antonio, TX: The Psychological Corporation; 2003. 
19. Beery K. Beery VMI-Administration, Scoring and Teaching Manual. Pearson: Bloomington, 2010.

20. Sortor JM, Kulp MT. Are the results of the Beery-Buktenica Developmental Test of Visual-Motor Integration and its subtests related to achievement test scores? Optometry Vision Sci 2003;80:758-63.

21. Manly T, Robertson IH, Anderson V, et al. TEA-Ch Test of Everyday Attention for Children. Pearson Assessment 1991: 1065-81.

22. Cserjesi R, Van Braeckel K, Butcher PR, et al. Functioning of 7-year-old children born at 32 to 35 weeks' gestational age. Pediatrics 2012;130: E838-46.

23. Talge NM, Holzman C, Wang J, et al. Late-preterm birth and its association with cognitive and socioemotional outcomes at 6 years of age. Pediatrics 2010;126:1124.

24. Anderson JW, Johnstone BM, Remley DT. Breast-feeding and cognitive development: a meta-analysis. Am J Clin Nutr 1999;70:525-35.
25. Geldof CJ, van Wassenaer AG, de Kieviet JF, et al. Visual perception and visual-motor integration in very preterm and/or very low birth weight children: a meta-analysis. Res Dev Disabil 2012;33:726-36.

26. van Soelen IL, Brouwer RM, Peper JS, et al. Effects of gestational age and birth weight on brain volumes in healthy 9 year-old children. J Pediatr 2010;156:896-901.

27. Peterson BS, Vohr B, Staib LH, et al. Regional brain volume abnormalities and long-term cognitive outcome in preterm infants. JAMA 2000;284: 1939-47.

28. Domellof E, Johansson AM, Farooqi A, et al. Relations among upper-limb movement organization and cognitive function at school age in children born preterm. J Dev Behav Pediatr 2013;34:344-52.

29. Løhaugen GCC, Østgård HF, Andreassen S, et al. Small for gestational age and intrauterine growth restriction decreases cognitive function in young adults. J Pediatr 2013;163:447-53 and 441. 